\title{
Diagnostic $X$ de la zone proche injecteur d'un jet cryogénique d'azote sous haute pression
}

\author{
B. Métay, E. Robert, R. Viladrosa, C. Cachoncinlle, J.M. Pouvesle, \\ W. Mayer ${ }^{1}$ et G. Schneider ${ }^{1}$
}

GREMI, IPO, CNRS, Université d'Orléans, 14 rue d'Issoudun, BP. 6744, 45067 Orléans cedex 2, France

${ }^{1}$ German Aerospace Center, DLR Lampoldshausen, 74239 Hardthausen, Allemagne

\begin{abstract}
Résumé: Une source impulsionnelle de rayons $\mathrm{X}$, compacte produisant des photons d'énergie comprise entre 5 et $50 \mathrm{keV}$, a été spécialement conçue au GREMI afin de pouvoir caractériser l'écoulement proche d'injecteurs cryogéniques $(20$ premiers $\mathrm{mm}$ ) sur l'installations M51 du DLR à Lampoldhausen à des pressions comprises entre 10 et 60 bars et des températures allant de 100 à $130 \mathrm{~K}$. Pour la première fois, des radiographies d'un jet cryogénique d'azote pur sous haute pression en sortie d'injecteur ont été réalisées sans ajout de traceur. Les données obtenues (cônes d'expansion, densité ) ont pu être comparées à celles mesurées par d'autres systèmes (ombroscopie, thermocouple) et présentent la radiographie comme particulièrement sensible et bien adaptée à ce genre de problématique.
\end{abstract}

\section{INTRODUCTION}

La tendance actuelle à augmenter la capacité de charge des lanceurs aérospatiaux impose une constante amélioration de leurs propulseurs et notamment des propulseurs cryogéniques de type Vulcain. La phénoménologie complexe au sein de ce réacteur nécessite de simplifier l'étude de chaque élément de base, et plus particulièrement l'injecteur. C'est dans cette optique que le Deutsches zentrum für Luft- und Raumfarht effectue depuis de nombreuses années une partie de ses études sur l'écoulement et la combustion en sortie d'un injecteur unique de jets cryogéniques se détendant dans une ambiance à haute pression. Les résultats obtenus ont permis de connaître une grande partie du comportement du jet ( répartition des densités, champs des vitesses ...) mais la zone proche de l'injecteur, très dense, restait interdite aux analyses optiques classiques [1]. Des tests en radiographie ayant déjà été effectués sur des jets denses dopés [2], nous avons développé une nouvelle source impulsionnelle de rayons X, rapide ( environ $50 \mathrm{~ns}$ ), compacte, afin de pouvoir étudier cette zone du jet cryogénique d'azote pur sans ajout de traceur.

$\mathrm{Au}$ cours d'expériences réalisées sur le banc d'essais M51 du DLR, ce Flash X, spécialement dédié à la production de rayons $X$ de faible énergie $(\sim 9 \mathrm{KeV})$, nous a permis d'effectuer des clichés radiographiques de la zone proche de l'injecteur $(<x / d=10$, avec $\mathrm{x}$ distance sur l'axe longitudinal du jet et d diamètre du jet) pour des pressions d'azote ambiant de 10 à 60 bars et des températures de jet d'azote liquide de températures comprises entre 100 à $130^{\circ} \mathrm{K}$.

\section{MONTAGE EXPERIMENTAL}

Les expériences ont été réalisées sur le banc d'essais cryogénique M51 du DLR à Lampoldhausen en Allemagne en utilisant une source flash de rayonnement $X$ spécifique développée au GREMI. L'ensemble du dispositif expérimental comprenant la chambre haute pression équipée d'un injecteur modèle ainsi que la source $\mathrm{X}$ est montré dans la photographie de la figure 1. La chambre haute pression, équipée de quatre fenêtres de visées peut être pressurisée jusqu'à 60 bars avec de l'azote à la température ambiante dans le cas présent. Elle contient un injecteur cryogénique permettant d'introduire de l'azote liquide à des vitesses comprises entre 1 et $10 \mathrm{~m} / \mathrm{s}$ à des températures bien contrôlées, entre 100 et $130 \mathrm{~K}$ dans le cadre de ce travail. Sur la figure 1 , on peut voir le flash $\mathrm{X}$ portable spécialement développé pour être adapté, à la fois, à l'environnement de la chambre haute pression (caractéristiques 
géométriques, fenêtre spéciale ) et au milieu à caractériser ( énergie des photons entre 5 et $50 \mathrm{KeV}$ ). Il est composé de deux ensembles distincts, une partie alimentation et pompage, et une partie source, déportée, comprenant le système de mise en forme de l'impulsion haute tension et la diode $\mathrm{X}$. Le principe de fonctionnement de ce flash est très similaire à d'autres développés précédemment au GREMI [3-4]. L'impulsion X est obtenue par décharge haute tension, en configuration anode pointe / cathode disque, dans la diode à une pression inférieure à $5.10^{-2}$ mbar. Pour une tension de charge de $28 \mathrm{kV}$, les doses émises sont de $0.3 \mathrm{mrad}$ à $1 \mathrm{~m}$ dans les impulsions de 50 ns FWHM. La très courte durée des impulsions $\mathrm{X}$ par rapport au temps caractéristique d'expansion du jet permet de totalement négliger le flou de bougé dans l'analyse des radiographies obtenues. Il faut par contre tenir compte de la taille de la source ( $\varnothing \sim 300 \mu \mathrm{m}$ ) et ses fluctuations en position dans le cas d'images obtenues sur plusieurs tirs.

Les photons $\mathrm{X}$ pénètrent dans la chambre de $12 \mathrm{~cm}$ de largeur, maintenue sous pression d'azote ( $10-60$ bars $)$ à travers une fenêtre spéciale équipée d'un hublot $(\varnothing 9 \mathrm{~mm}$ ) en Mylarß de $320 \mu \mathrm{m}$ d'épaisseur. Ils interagissent avec le gaz ambiant et le jet cryogénique ( température comprise entre 100 et $130 \mathrm{~K}$ ) d'un diamètre de $2.2 \mathrm{~mm}$ à la sortie de l'injecteur placé au centre de la cellule. La radiographie est obtenue en reprenant l'image par ICCD (Intensified Charge Coupled Device) d'un scintillateur ACS (CsI sur une couche de carbone amorphe) placé dans la chambre à une distance suffisante du jet pour ne pas perturber l'écoulement. Les mesures sont réalisées sur les deux premiers $\mathrm{cm}$ après la sortie de l'injecteur dans la zone la plus dense du jet difficilement accessible aux diagnostics optiques. Compte tenu des milieux en présence, on obtient un contraste suffisant sur l'épaisseur du jet entre celui-ci et le gaz ambiant pour des photons $\mathrm{X}$ dans la gamme d'énergie allant de 8 à $20 \mathrm{keV}$, gamme d'énergie pour laquelle le flash a été optimisé ( raies caractéristiques du matériau de l'anode en tungstène $L \alpha$ et $L_{\beta}$ entre 8 et $10 \mathrm{keV}$ et continu de Bremsstrahlung avec un maximum entre 10 et $15 \mathrm{keV}$.

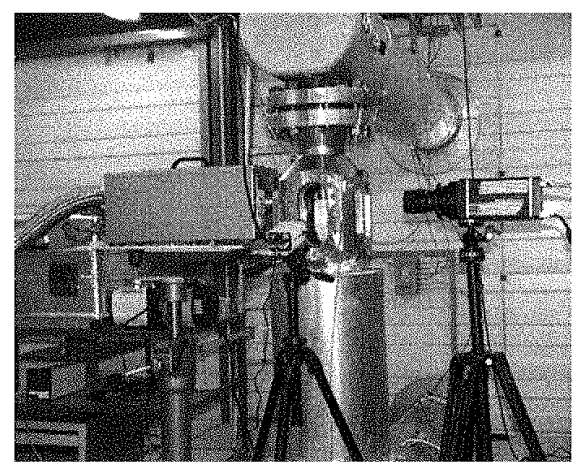

figure 1: photographie d'ensemble du dispositif sur l'installation M51 du DLR de Lampholdhausen

\section{CALCUL DE LA DENSITE}

L'absorption des photons $\mathrm{X}$ traversant un milieu est lié à sa densité par la loi de Beer-Lambert ( équation 1 ). En supposant que le jet présente une symétrie cylindrique, hypothèse a priori justifiée dans la zone proche de l'injecteur, on peut remonter à la variation de densité suivant le rayon si le coefficient d'atténuation massique $\sigma$ et le spectre de la source sont connus.

Un algorithme, construit sur la définition de couches concentriques d'isodensité au sein du jet ( voir figure 2 ), a été développé ( thèse L.Huré [5] ) permettant une reconstruction du jet à partir de l'analyse de l'image radiographique. L'épaisseur de chaque couche est définie à partir de la taille des pixels du détecteur suivant des considérations purement géométriques en tenant compte des distances source-jet et jet-détecteur. Le profil de densité est reconstitué de proche en proche en partant du bord vers le centre du jet en calculant l'épaisseur du milieu traversé pour chaque couche et la densité par inversion de la loi de Beer-Lambert. Le spectre de la source a été obtenu par mesure de filtres de Ross et par échelles de transmission. Sa 
modification le long du parcours dans le milieu gazeux, due à la variation de $\sigma$ (données NIST [6] ) en fonction de l'énergie des photons, est bien sûr prise en compte dans les calculs.

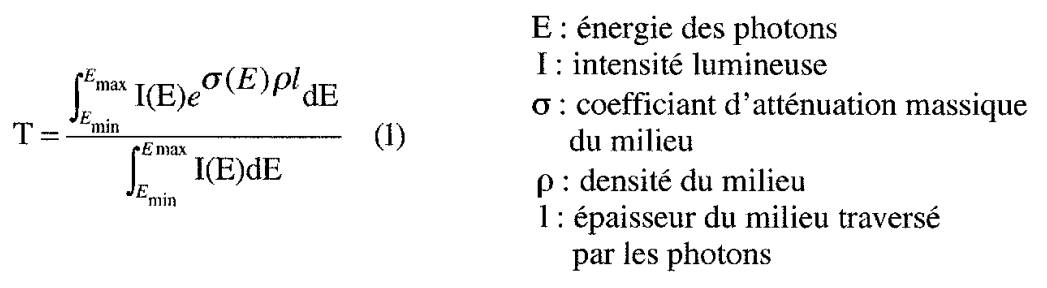

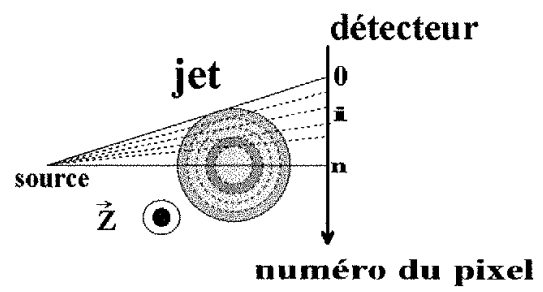

figure 2 : schéma de principe pour la définition des anneaux d'isodensité

\section{RESULTATS EXPERIMENTAUX ET ANALYSE}

Des exemples de radiographies obtenues dans différentes conditions expérimentales sont données figure 3. Quelles que soient les valeurs des pressions de gaz ambiant (10, 40,60 bars ) et les températures de jet ( 100,120 et $130 \mathrm{~K}$ ), des contrastes suffisants ont été obtenus pour permettre une analyse des images suivant la méthode décrite au paragraphe précédent. Nous avons pu mettre en évidence dans des conditions extrêmes, par exemple un jet à $120 \mathrm{~K}$ dans une atmosphère de 10 bars d'azote, correspondant à un jet de $0.032 \mathrm{~g} / \mathrm{cm}^{3}$ dans une ambiance à $0.012 \mathrm{~g} / \mathrm{cm}^{3}$, en se rappelant que le diamètre du jet est de seulement $2.2 \mathrm{~mm}$. Les radiographies ont été effectuées soit sur un seul tir, soit en moyennant sur 200 tirs, ce dernier cas permettant en effet de comparer directement les résultats obtenus par d'autres diagnostics faisant appel à des mesures moyennées.

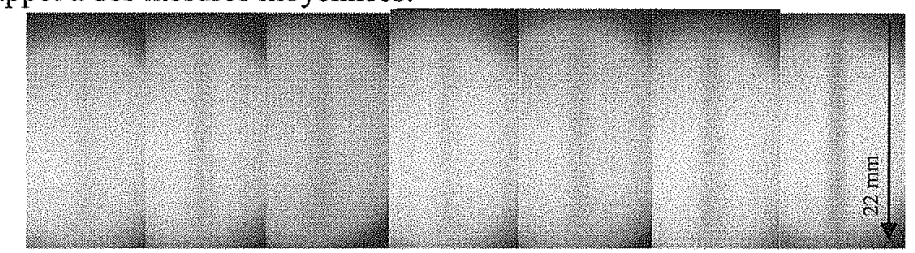

figure 3 : Radiographies des jets d'azotes pour les conditions suivantes ( de gauche à droite ): 60 bars à 130,120 et $100 \mathrm{~K}, 40$ bars à 130,120 et $100 \mathrm{~K}, 10$ bars à $100 \mathrm{~K}$

Dans le deuxième cas, l'analyse des images doit tenir compte du flou de bougé induit par de petites variations de position du point source dans l'espace inter-électrodes. L'image est alors traitée comme résultant d'une source équivalente de plus grande dimension $\varnothing$ de 1 à $2 \mathrm{~mm}$ suivant les cas, mesurés à partir d'une référence fixe constituée d'un fil calibré se trouvant dans le champ de l'image ). Pour cette première série d'expériences, constituant essentiellement en une démonstration de faisabilité, les modifications sur le spectre et la transmission pouvant être dues à des mouvements convectifs avec des variations significatives 
de la température dans le gaz ambiant autour du jet n'ont pas été prises en compte ( une cartographie de température dans la chambre haute pression est en cours au DLR ). De manière à en partie s'affranchir de ces problèmes, un point référence de densité pour l'ensemble des mesures a été déterminé à partir de l'absorption au centre du jet au ras de l'injecteur pour une pression de 40 bars avec un jet de $100 \mathrm{~K}$. Les profils de densités obtenus à 40 bars pour trois températures différentes sont représentés figure 4 .

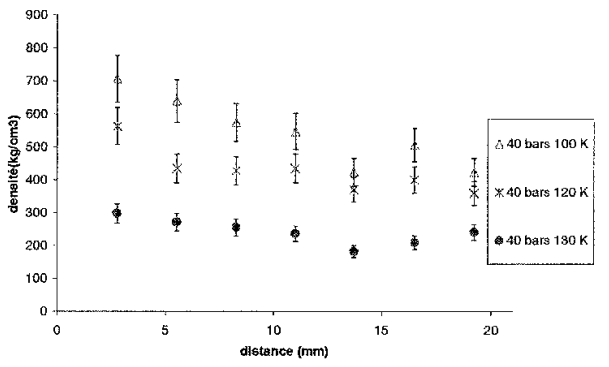

figure 4 : évolution de la densité le long de l'axe du jet pour différentes conditions de température à une pression de 40 bars, en fonction de la distance à la sortie de l'injecteur

\section{CONCLUSION}

La faisabilité de la radiographie de jets cryogéniques non dopés dans une atmosphère à haute pression dans des conditions de faibles contrastes a été démontrée. De nouvelles campagnes d'expériences vont être menées au DLR en réalisant soit des radiographies en monocoup soit des séries de radiographies avec un moyennage après traitement des images avec un recalage de celle-ci en fonction de la position exacte du point source. Cela permettra de limiter les problèmes inhérents aux légères fluctuations de position de la décharge dans l'espace interélectrodes. Des études sont en cours au DLR pour caractériser la recirculation du gaz autour du jet. Ces nouvelles études permettront d'améliorer la sensibilité de ce nouvel outil de diagnostics et d'étendre son utilisation à des conditions d'injection encore plus contraignantes en combustion.

\section{Références}

[1] W.Mayer, A. Schik, C. Schweitzer, M. Schäffler,

Injection and mixing processes in high pressure LOX/GH2 rocket combustor ( 32 nd AIAA/ASME/SAE/ASEE Joint Propulsion Conference, 1996 ) AIAA 96-2620

[2] Y.Yue, C.F Powell, R.Poola,J.Wang, J.K Schaller, Quantitative measurement of diesel fuel spray characteristics in the near-nozzle region using x-ray absorption (Atomization and spray 2001) vol.11, pp 471-490.

[3] J.M. Pouvesle, C.cachoncinlle, R.Viladrosa,E.Robert et A.Khacef , Nucl. Instrum. Meth. Phys. B, $\underline{113}, 134$ (1996)

[4] J.Geiswiller, E.Robert, L.Huré, C.Cachoncinlle, and J.M.Pouvesle, Meas. Sci. Technol.,9, 1537 (1998)

[5] L. Huré, Etude de l'application de la fluorescence induite par flash de rayonnement X et de la radiographie «éclair » au diagnostic et à la carctérisation de milieux mono- et diphasiques en écoulement, thèse univ. Orléans, 2000

[6] NIST : http://physics.nist.gov/PhysRefData/contents.html 